\title{
Impaired Activation of Adipocyte Lipolysis in Familial Combined Hyperlipidemia
}

\author{
Signy Reynisdottir, Mats Eriksson, Bo Angelin, and Peter Arner \\ Department of Medicine, Research Center, and Molecular Nutrition Unit, Center for Nutrition and Toxicology, Karolinska Institute at \\ Huddinge University Hospital, S-14186 Huddinge, Sweden
}

\begin{abstract}
The pathophysiology of familial combined hyperlipidemia (FCHL) is unknown, but altered lipid turnover in peripheral tissues as well as hepatic overproduction of apolipoprotein $B$ have been suggested as possible causes. In the present study, we explored whether a change in triglyceride breakdown by lipolysis in fat cells is present in FCHL. Lipolysis activation by catecholamines was examined in isolated subcutaneous adipocytes from 10 patients with FCHL and 22 healthy control subjects.

Lipolysis rate was linear for at least $3 \mathrm{~h}$ in both groups. However, a marked $(\sim 65 \%)$ decrease in the lipolytic response to noradrenaline was found in FCHL. This was also true when lipolysis was maximally stimulated at the receptor level with isoprenaline (nonselective $\beta$-adrenergic agonist), at the adenylyl cyclase level with forskolin, or at the level of the protein kinase hormone-sensitive lipase complex with dibutyryl cAMP. The maximum enzymatic activity of hormone-sensitive lipase was decreased by $\sim 40 \%$ in FCHL. On the other hand, the lipolytic sensitivity of $\alpha_{2}^{-}, \beta_{1-}^{-}$, and $\beta_{2}$-adrenoceptors was normal in this condition, as was the number and affinity of $\boldsymbol{\beta}_{1}$ - and $\boldsymbol{\beta}_{2}$-adrenoceptors. Variations in the maximum lipolysis rate correlated significantly with the variations in hormone-sensitive lipase activity in the whole material, and with the serum values for triglycerides, HDL cholesterol and apoB lipoprotein within the control group, but the serum triglyceride values in FCHL were higher than this correlation predicted. In conclusion, the data demonstrate a marked resistance to the lipolytic effect of catecholamines in fat cells from patients with FCHL, in spite of normal adrenoceptor function. The lipolytic defect appears predominantly to be due to a defect in hormonesensitive lipase, and may be of importance in the pathophysiology of FCHL. (J. Clin. Invest. 1995. 95:2161-2169.) Key words: hormone-sensitive lipase - catecholamines • adrenoceptors - cAMP
\end{abstract}

Address correspondence to Peter Arner, Department of Medicine, Huddinge University Hospital, S-141 86 Huddinge, Sweden. FAX: 46-8746-4133.

Received for publication 5 May 1994 and in revised form 14 December 1994.

1. Abbreviations used in this paper: BMI, body mass index; dcAMP, dibutyryl cAMP; FCHL, familial combined hyperlipidemia; HSL, hormone-sensitive lipase.

J. Clin. Invest.

(C) The American Society for Clinical Investigation, Inc.

$0021-9738 / 95 / 05 / 2161 / 09 \$ 2.00$

Volume 95, May 1995, 2161-2169

\section{Introduction}

Familial combined hyperlipidemia $(\mathrm{FCHL})^{1}$ is the most common familial form of hyperlipidemia in young survivors of myocardial infarction, and may cause at least $10 \%$ of premature coronary heart disease (1-5). Manifest hyperlipidemia in FCHL usually does not occur before the age of $20 \mathrm{yr}$ (3), and no specific biological marker has yet been identified (5). Instead, diagnosis is generally based on family studies: if multiple lipoprotein phenotypes (IIA, IIB, and IV) are found among the relatives of a family, and also among a single individual studied on different occasions, FCHL is considered to be present $(5,6)$. Studies based upon such criteria have demonstrated metabolic differences between FCHL and monogenic familial hypertriglyceridemia (5-10), and have also suggested possible genetic linkage of FHCL to the lipoprotein lipase gene (11), and to the apo AI-CIII-AIV gene cluster $(12,13)$. However, no definite metabolic defect has yet been identified in FCHL, although overproduction of VLDL apo B is a frequent finding (7-9). In addition, chylomicron remnant clearance is delayed in FCHL, and prolonged elevations of plasma FFA occur postprandially $(14,15)$. The fact that hypertriglyceridemia alone was the earliest manifestation in affected children (1) has focused on the possible role of disturbances in triglyceride and FFA metabolism in the pathogenesis of FCHL (15).

Other causes for FCHL that have been proposed include abnormal lipoprotein metabolism due to altered lipid turnover in peripheral tissues $(5,16)$. In this case, adipose tissue is of particular interest, since fat cells are the main triglyceride reservoir of the body and also have a large capacity to store cholesterol (17). It has been suggested that stimulation of FFA incorporation into triglycerides in fat cells by acylation-stimulating protein, a potent activator of triglyceride synthesis, is inhibited in patients with hyperapobetalipoproteinemia $(18,19)$, a condition apparently related to FCHL (20). A partial lipoprotein lipase deficiency has been demonstrated in some families with FCHL (11), which further indicates a decreased lipid synthesis in the adipocytes. The possible role of abnormal breakdown of triglycerides by lipolysis in fat cells has to our knowledge not been studied in this disorder. It is logical to assume that if the turnover rate of lipids is decreased in fat cells, a diminished synthesis rate of triglycerides should be counterbalanced by a decrease in the triglyceride hydrolysis rate.

In humans, catecholamines are the most important lipolytic hormones (21). They bind to $\beta$-adrenoreceptors, thus activating adenylyl cyclase and increasing intracellular cAMP which in turn activates the hormone-sensitive lipase (HSL). The latter catalyzes the breakdown of triglycerides to glycerol and FFA. Binding to counterregulatory $\alpha_{2}$-adrenoceptors causes inhibition of adenylyl cyclase and thus of lipolysis. The activation of HSL is rate limiting for lipolysis, while the sensitivity to catecholamine action is modulated at the receptor level.

In the present study, the existence of a possible defect in lipolysis regulation was investigated in subcutaneous adipocytes 
Table I. Clinical Characteristics of Patients with FCHL

\begin{tabular}{|c|c|c|c|c|c|c|c|c|c|}
\hline No. & Age & $\begin{array}{l}\text { Body mass } \\
\text { index }\end{array}$ & $\begin{array}{c}\text { Total } \\
\text { cholesterol }\end{array}$ & $\begin{array}{c}\text { HDL } \\
\text { cholesterol }\end{array}$ & $\begin{array}{c}\text { Total } \\
\text { triglycerides }\end{array}$ & Apo B & Apo AI & $\begin{array}{l}\text { Observed lipoprotein } \\
\text { phenotypes }\end{array}$ & $\begin{array}{l}\text { Coronary heart } \\
\text { disease }\end{array}$ \\
\hline & $y r$ & $\mathrm{~kg} / \mathrm{m}^{2}$ & mmolliter & mmolliter & mmolliter & g/iter & g/iter & & \\
\hline 1 & 48 & 23.8 & 7.2 & 1.2 & 3.6 & 2.0 & 1.41 & IIB-IV-N & - \\
\hline 2 & 33 & 24.8 & 8.2 & 0.8 & 5.5 & 1.63 & 0.99 & IIA-IIB-IV & - \\
\hline 3 & 32 & 23.1 & 7.3 & 0.9 & 2.9 & 1.87 & 1.33 & IIB-IIA & - \\
\hline 4 & 40 & 27.5 & 6.3 & 1.2 & 1.3 & 1.18 & 1.50 & IIA-IIB-IV & + \\
\hline 5 & 46 & 24.5 & 5.2 & 1.2 & 1.4 & 1.05 & 1.25 & IV-IIB & + \\
\hline 6 & 61 & 28.4 & 6.5 & 1.4 & 2.7 & 1.07 & 1.38 & IIB-IV & + \\
\hline 7 & 42 & 23.0 & 6.6 & 0.8 & 3.0 & 1.71 & 1.21 & IIB-IV & - \\
\hline 8 & 39 & 27.1 & 9.1 & 1.0 & 3.3 & 1.63 & 1.30 & IIA-N-IIB & - \\
\hline 9 & 47 & 26.9 & 9.9 & 0.9 & 3.7 & 2.57 & 1.13 & IIA-IIB-IV & - \\
\hline 10 & 49 & 24.8 & 6.8 & 0.6 & 6.2 & 1.71 & 1.09 & IV-IIB & - \\
\hline
\end{tabular}

The numbers correspond to the number of each proband in the pedigrees in Fig. 1 . The serum lipid values are from the day of the study. The different lipoprotein phenotypes were observed during clinical follow-up for several years. The diagnosis of coronary heart disease is based on previous myocardial infarction, coronary heart surgery.

from men with FCHL. Isolated fat cells were incubated with agents that act on various defined steps in the lipolytic cascade, from the adrenoceptors to the activation of HSL. The results suggest that FCHL is associated with a defective activation of lipolysis at the level of HSL.

\section{Methods}

Subjects, experimental procedure. The study included 10 nonobese male patients with FCHL (Table I), who had been followed as outpatients for several years. The diagnosis was based on the finding of hyperlipidemia in first degree relatives, among which at least one had a lipoprotein phenotype that differed from that of the proband (22). In addition, variable lipoprotein phenotypes had generally been observed during the clinical follow-up of these patients. The pedigrees showing the information available at the time of the study are found in Fig. 1. Of a total of 76 known probands, 37 had hyperlipidemia. This is consistent with a hereditary hyperlipidemia with a dominant trait, as has been described in FCHL (1-5). In 21 subjects, information on blood lipid levels could not be obtained. 19 subjects had manifest coronary heart disease, i.e., they had had myocardial infarction or undergone coronary vessel surgery because of angina pectoris.

A patient or relative was considered to be hyperlipidemic if the serum cholesterol or triglyceride level exceeded the 90th percentile of the value in the general population, adjusted for age and sex. The lipoprotein phenotyping was based on the levels of total cholesterol and triglyceride. Type III hyperlipidemia was excluded by agarose gel electrophoresis.

The patients were in stable metabolic condition and secondary hyperlipidemia had been excluded. They had all been instructed to follow a diet corresponding to American Heart Association step I. There was no evidence of intestinal, liver, renal, or thyroid disease, nor had any subject had myocardial infarction less than 6 mo before the study. Five patients $(1-3,8$, and 10 in Fig. 1 and Table $I)$ were without any type of medication, whereas five patients (No. 4-7 and 9) were on stable treatment with lipid-lowering agents (fibrates) since at least 6 mo.

22 healthy, nonobese, and drug-free volunteers comprised a control group. The two groups were matched for body mass index (BMI) and age. The control subjects were normolipidemic, with the exception of one subject who had a slightly elevated serum triglyceride value (2.5 $\mathrm{mmol} /$ liter ). In this case, however, any form of familial dyslipidemia could be excluded after analysis of blood samples from all first degree relatives. Clinical data comparing the two groups are shown in Table
II. All undertook light (walking) or moderate exercise at regular intervals, non was sedentary.

The subjects were examined at 8:00 a.m. after an overnight fast. First, venous samples for analysis of hormones, metabolites, and serum
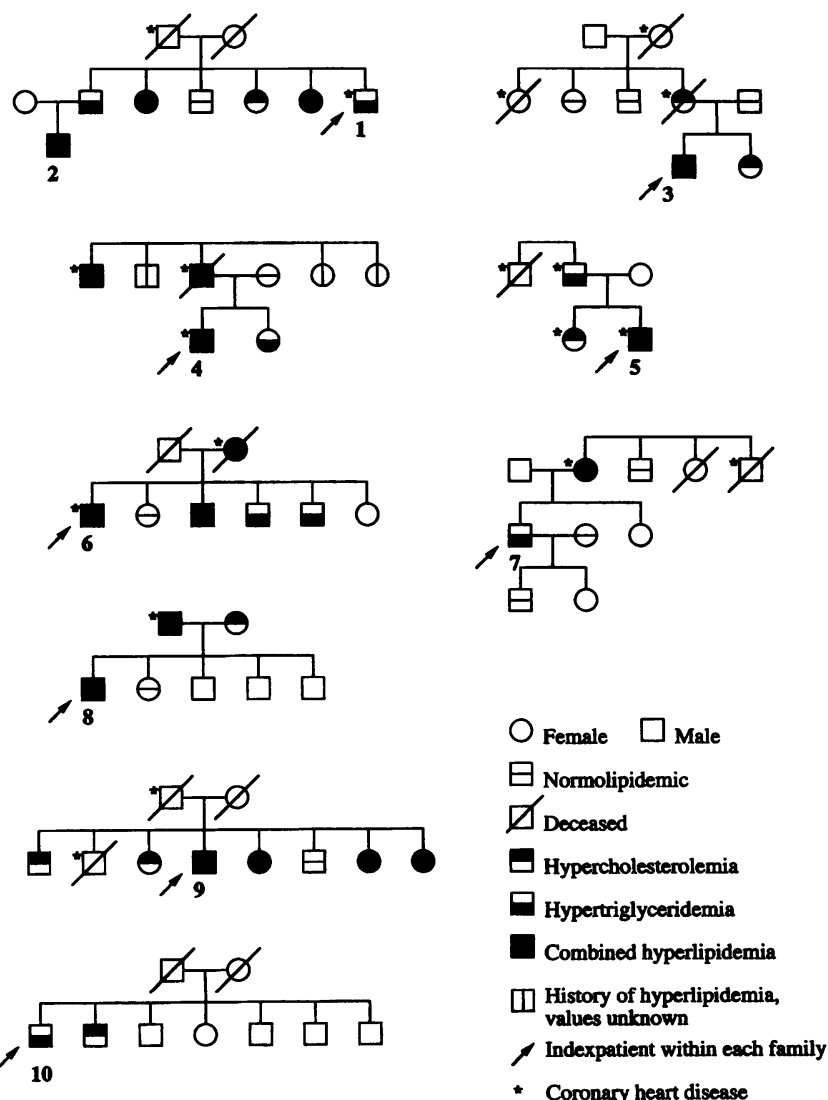

Figure 1. Pedigrees of 10 patients with FCHL. The proband is indicated with a number which corresponds to the number in Table I. * Indicates subjects with established coronary heart disease (previous coronary heart surgery and/or myocardial infarction). 
lipid profiles were collected. Blood pressure was measured in the supine position. Second, a subcutaneous fat biopsy, $\sim 2-4 \mathrm{~g}$, was surgically removed from the paraumbilical region under local anesthesia as described (23). The tissue was placed in saline solution and immediately transported to the laboratory.

All subjects had given their informed consent before entering the study, which had been approved of by the ethics committee of the Karolinska Institute, Huddinge, Sweden.

Determination of hormones and metabolites. Plasma insulin and catecholamines, as well as lipoprotein levels, were determined as described $(24,25)$. Routine laboratory analyses were performed at the hospital's department of clinical chemistry.

Isolation of fat cells and determination of fat cell size. Isolated fat cells were prepared through incubation in collagenase according to Rodbell (26). Fat cell size was determined as follows: an aliquot of cells, suspended in an albumin buffer solution, was placed on a glass slide and the diameter of 100 cells was determined by examination with a microscope (Carl Zeiss, Inc., Thornwood, NY) equipped with a caliper scale. Mean fat cell volume and weight were then calculated according to the methods developed by Hirsch and Gallian (27). The total lipid content in each incubation was determined gravimetrically after organic extraction. The number of fat cells in each incubation can be estimated by dividing the total lipid weight with the mean cell weight. This method was compared with a more tedious method (28), where the number of fat cells is directly determined by counting cells in small aliquots of appropriately diluted fat cell suspensions. The correlation between the two methods was excellent $(r=0.97 ; n=10)$.

Lipolysis experiments. This assay has previously been described in detail $(17,24)$. A diluted suspension of fat cells was incubated at $37^{\circ} \mathrm{C}$ in duplicate samples, with or without increasing concentrations of the nonselective natural catecholamine noradrenaline, the nonselective $\beta$ adrenergic agonist isoprenaline, the $\beta_{2}$-selective agonist terbutaline, the $\beta_{1}$-selective agonist dobutamine, the $\alpha_{2}$ adrenergic agonist clonidine, forskolin which stimulates adenylyl cyclase, and dibutyryl cAMP (dcAMP) which activates HSL. Glycerol release to the incubation medium was used as an index of the lipolysis rate. After $2 \mathrm{~h}$ incubation, an aliquot of the incubation medium was removed for analysis of glycerol content using an automated bioluminescence assay (29).

All the agonists caused a dose-dependent increase or inhibition of glycerol release, reaching a plateau at the highest agonist concentrations. The sensitivity to agonist action, defined as the concentration of each agonist giving half maximum effect $\left(E D_{50}\right)$, was determined by linear regression analysis after log-logit transformation of each individual concentration response curve. The responsiveness to each agonist was determined as the maximum stimulated lipolysis rate. The lipolysis rates in the presence or absence of agonist were related to the number of incubated cells.

As shown previously (29), the activation of lipolysis under these conditions is linear with time in healthy subjects. In a separate set of methodological experiments, a second fat biopsy, $\sim 1.5 \mathrm{~g}$, was obtained from two of the FCHL patients and two control subjects to determine whether this was true also for these patients. Isolated fat cells were incubated as described above with maximum effective concentrations of noradrenaline, isoprenaline, forskolin, and dibutyryl cAMP, for 30, $60,90,120$, and $180 \mathrm{~min}$, respectively, whereafter aliquots were taken for glycerol analysis.

Assay of hormone-sensitive lipase activity. This assay was performed as described by Fredriksson et al. (30) with the modifications for handling of small samples of human adipose tissue exactly as described by Frayn et al. (31). First, pieces of adipose tissue $(\sim 100 \mathrm{mg})$, which had been stored in liquid nitrogen, were homogenized at $4^{\circ} \mathrm{C}$ in $2.0 \mathrm{ml}$ of a buffer containing $0.25 \mathrm{~mol} /$ liter sucrose, $1 \mathrm{mmol} /$ liter EDTA, $1 \mathrm{mmol} / \mathrm{liter}$ DTT, and $20 \mu \mathrm{mol} / \mathrm{liter}$ each of the protease inhibitors antipain and leupeptin. The homogenate was then centrifuged at $100,000 \mathrm{~g}$ at $4^{\circ} \mathrm{C}$ for $45 \mathrm{~min}$. The fat cake was removed, and the fatfree infranatant recovered for analysis of HSL activity using 1(3)$\left[{ }^{3} \mathrm{H}\right]$ oleoyl-2-O-oleylglycerol as substrate $(30)$. The substrate was obtained from the same source of production (Dept. of Medical and Physi- ological Chemistry, Lund University, Lund, Sweden) as in the original methodological studies $(30,31)$. All samples were incubated in triplicate at $37^{\circ} \mathrm{C}$ for $30 \mathrm{~min}$ on one occasion. The substrate for HSL has only one hydrolyzable ester bond and is therefore not a substrate for monoacylglycerol lipase which is abundant in adipose tissue. Furthermore, at the present incubation conditions ( $\mathrm{pH} 7.0$ and no apo CII present) lipoprotein lipase activity is negligible (30). Since the phosphorylated and dephosphorylated forms of the enzyme have the same activity towards this substrate, only the total amount of activable enzyme in the sample is measured (31). Hormone-sensitive lipase hydrolyzes tri- and diacylglycerol at the relative rates of 1:10 (30). Therefore, the sensitivity of the assay is enhanced by the use of a diacylglycerol analog as substrate. $1 \mathrm{U}$ of enzyme activity equals $1 \mu \mathrm{mol}$ of fatty acid produced per minute at $37^{\circ} \mathrm{C}$. Enzyme activity was related to the total protein concentration of the sample which was measured using a commercial protein assay reagent (BCA; Pierce, Rockford, IL). The enzyme activity and total protein concentration of the fat-free infranatant were proportional to the wet weight of the tissue specimen when multiple samples from one subject were analyzed $(r=0.95 ; P<0.005)$. The within run coefficient of variation was $7 \%$. The intraindividual coefficient of variation, examined by multiple samples of adipose tissue from one subject was $11 \%$.

Radioligand binding assays. The radioligand binding studies with isolated fat cells have been described previously (24). Briefly, the cells were incubated with the nonselective $\beta_{1}-\beta_{2}$-radioligand ${ }^{125} \mathrm{I}$-cyanopindolol for $60 \mathrm{~min}$ at $37^{\circ} \mathrm{C}$, and cell-bound radioactivity was measured.

Saturation experiments were performed in duplicate with increasing concentrations of the radioligand (10-750 pmol/liter). Nonspecific binding was measured in the presence of propranolol $\left(10^{-5} \mathrm{~mol} / \mathrm{liter}\right)$, and was $\sim 30 \%$ at low and $\sim 45 \%$ at high radioligand concentrations. The total number of $\beta$-adrenoceptor binding sites and the $K_{\mathrm{d}}$ values for the radioligand were determined through linear regression analysis of Scatchard plots (32).

Displacement experiments were performed with $100 \mathrm{pmol} /$ liter of the radioligand, which was displaced by 12 increasing concentrations of the $\beta_{2}$-selective antagonist ICI $118,551\left(0,10^{-11}-10^{-4} \mathrm{~mol} / \mathrm{liter}\right)$. Nonspecific binding, defined as the binding at $10^{-4} \mathrm{~mol} / \mathrm{liter}$, was $\sim 30 \%$. The experiments gave shallow biphasic displacement curves. A nonlinear least squares regression analysis was performed to determine the proportions of high $\left(\beta_{2}\right)$ and low affinity $\left(\beta_{1}\right)$ binding sites.

Finally, the results of the saturation and displacement experiments were taken together to determine the total number of $\beta_{1}$ - and $\beta_{2}$-adrenoceptors, respectively. The concentrations of the radioligand used are too low to identify $\beta_{3}$-adrenoceptors, for which ${ }^{125} \mathrm{I}$-cyanopindolol has low affinity (33).

Drugs and chemicals. BSA (fraction V, lot 63F-0748), Clostridium histiolyticum collagenase type I, propranolol, forskolin, dibutyryl cAMP, glycerol kinase from Escherichia coli (G4509), antipain, and leupeptin were obtained from Sigma Chemical Co. (St. Louis, MO). (-)-Isoprenaline was from Hässle (Mölndal, Sweden), terbutaline from Draco (Lund, Sweden), dobutamine from Eli Lilly and Co. (Indianapolis, IN), and ICI 118,551 from Cambridge Research Biochemicals Ltd. (Cheshire, United Kingdom). ATP monitoring reagent containing firefly luciferase was obtained from LKB Wallac (Turku, Finland), and ${ }^{125} \mathrm{I}$ cyanopindolol from New England Nuclear (Boston, MA). ${ }^{3} \mathrm{H}$-monoacylmonoalkylglycerol was from Dr. Cecilia Holm, Dept. of Medical and Physiological Chemistry, Lund University, Lund, Sweden. All other chemicals were of the highest grade of purity commercially available. Collagenase and all ingredients in the incubation buffers were from the same batches throughout the study.

Statistical analysis. Student's two-tailed $t$ test or chi-square (with Yate's correction) was used for comparison of data between groups. In some cases simple or stepwise regression analysis was performed. All values are expressed as mean $\pm \mathrm{SEM}$. The values for $K_{\mathrm{d}}$ and $\mathrm{ED}_{50}$ were transformed into their logarithmic form before statistical evaluation. All statistics (except chi-square) were performed with the aid of a software statistical package (Stat View II; Abacus Concepts Inc., Berkeley, CA). 
Table II. Clinical Characteristics

\begin{tabular}{lccc}
\hline & $\begin{array}{c}\text { FCHL patients } \\
(n=10)\end{array}$ & $\begin{array}{c}\text { Control group } \\
(n=22)\end{array}$ & $P$ \\
\hline Age (yr) & $43.7 \pm 2.7$ & $48.7 \pm 3.7$ & NS \\
Smokers/nonsmokers & $2 / 8$ & $5 / 17$ & NS \\
BMI (kg/m $)$ & $25.4 \pm 0.6$ & $24.3 \pm 0.4$ & NS \\
W/H ratio & $0.998 \pm 0.012$ & $0.947 \pm 0.01$ & $<0.01$ \\
Cell volume (pl) & $497 \pm 51$ & $442 \pm 33$ & NS \\
S-cholesterol & & & \\
$\quad$ (mmol/liter) & $7.11 \pm 0.40$ & $5.68 \pm 0.20$ & $<0.001$ \\
S-triglycerides & & & \\
$\quad$ (mmol/liter) & $3.20 \pm 0.52$ & $1.21 \pm 0.10$ & $<0.0001$ \\
S-HDL-cholesterol & & & \\
$\quad$ (mmol/liter) & $1.0 \pm 0.08$ & $1.26 \pm 0.09$ & $<0.05$ \\
S-apo B (g/liter) & $1.64 \pm 0.15$ & $1.21 \pm 0.06$ & $<0.005$ \\
S-apo A I (g/liter) & $1.26 \pm 0.05$ & $1.42 \pm 0.06$ & NS \\
B-glucose (mmol/liter) & $5.4 \pm 0.2$ & $4.7 \pm 0.1$ & $<0.005$ \\
P-insulin (mU/ml) & $9.2 \pm 1.4$ & $7.2 \pm 1.3$ & NS \\
P-glycerol (mmol/liter) & $60.9 \pm 6.4$ & $57.9 \pm 4.3$ & NS \\
P-noradrenaline & & & \\
$\quad$ (nmol/liter) & $1.95 \pm 0.37$ & $2.02 \pm 0.27$ & NS \\
P-adrenaline & & & \\
$\quad$ (nmol/liter) & $0.19 \pm 0.04$ & $0.16 \pm 0.02$ & NS \\
& & & \\
\hline
\end{tabular}

The values are mean \pm SEM. They were compared using Student's $t$ test. S, serum; P, plasma; B, blood. NS, not significant. W/H, waist-hip.

\section{Results}

Clinical data on the patients and the controls are shown in Table II. The control subjects were selected to match the patients as well as possible in regards to age and BMI. Although the FCHL patients had a slightly higher waist-hip ratio, fat cell size was similar in the two groups. As expected, total serum cholesterol, triglycerides, and apo B levels were higher in the FCHL patients than in the control group, while serum HDL cholesterol levels were lower. However, there was no significant difference in serum apo AI levels. Blood glucose was higher in the patients, but plasma glycerol, catecholamine, and insulin levels did not differ significantly between the two groups.

In accordance with previous studies (29), the activation of lipolysis with noradrenaline, isoprenaline, forskolin, or dcAMP was linear with time for at least $3 \mathrm{~h}$ in healthy subjects (Fig. 2). This was also true for FCHL patients (Fig. 2).

The mean concentration reponse curves for lipolysis upon stimulation with different lipolytic agents are shown in Fig. 3. In regards to the nonselective natural catecholamine noradrenaline, a marked reduction in the lipolysis response was found in fat cells from the patients with FCHL as compared to the control group. This difference persisted at maximal as well as at submaximal effective concentrations of noradrenaline. The figure also shows the results of lipolysis stimulation with agonists acting at different levels in the lipolytic cascade. In FCHL, a marked reduction of the lipolytic response $(\sim 65 \%)$ was found whether lipolysis was stimulated nonselectively via $\beta$-adrenoceptors with isoprenaline, by activation of the adenylyl cyclase with forskolin, or by direct activation of the protein kinaseHSL complex with dcAMP. The individual values of the maximum lipolytic effect of each agonist are statistically compared in Table III. With noradrenaline, the maximum lipolytic rate was reduced by two-thirds in FCHL $(P<0.01)$. With the other agents, it was reduced by about half $(P<0.005)$. These differences in lipolysis rate between the groups persisted even when the data were calculated using lipid weight as denominator instead of cell number (data not shown). The basal rate of lipolysis (which was subtracted from the agonist-induced values) did not differ significantly between the groups. It was $7.0 \pm 1.7 \mu \mathrm{mol}$ of glycerol $/ 10^{7}$ cells in the FCHL patients, and $5.0 \pm 0.7 \mu \mathrm{mol} / 10^{7}$ cells in the control subjects.

The results presented so far indicated that the low noradrenaline response in the patients with FCHL was due to a postreceptor defect in lipolysis activation. To examine whether the low noradrenaline response to some degree was also due to altered adrenoceptor function, the lipolytic response to selective adrenergic agonists was examined. This was done using receptorsubtype-specific adrenergic agonists. Since we wanted to evaluate agonist sensitivity, which is a measure of receptor function, rather than the maximum effect, which above all reflects postreceptor events, the dose-response curves (Fig. 4) were expressed as percentage of the maximum rate. There were, however, no differences between the groups regarding the sensitivity to $\beta_{1}$ or $\beta_{2}$-adrenoceptor-mediated lipolysis or $\alpha_{2}$-adrenoceptor-mediated antilipolysis, the concentration response curves being practically superimposed. The individual $\mathrm{ED}_{50}$ values for each selective agonist, as well as for the nonselective agonists noradrenaline and isoprenaline, are shown in Table IV. No statistically significant differences were observed between the groups for any of the values. In regards to the maximum antilipolytic effect of clonidine, this agent caused an inhibition by $71 \pm 5 \%$ and $71 \pm 4 \%$ of the lipolysis rate in FCHL patients and controls, respectively.

The number of $\beta_{1-}$ and $\beta_{2}$-receptor binding sites was determined by radioligand binding. There was no significant difference between the two groups in the number of binding sites for either $\beta$-adrenoceptor subtype. The values for $\beta_{1}$-adrenoceptors were $0.48 \pm 0.11 \mathrm{pmol} / 10^{7}$ cells in FCHL and $0.58 \pm 0.09 \mathrm{pmol} /$ $10^{7}$ cells in the control group. The corresponding values for $\beta_{2-}$ adrenoceptors were $0.40 \pm 0.08$ and $0.60 \pm 0.10 \mathrm{pmol} / 10^{7}$ cells, respectively. The affinity of these receptor subtypes for the displacing drug, the $\beta_{2}$-selective antagonist ICI 118,551, did not differ between the groups either (data not shown).

The relationship between the serum lipid levels and the lipolysis defect was examined using simple regression analysis. In the control group, there was a linear relationship between the maximum lipolysis rate and the serum values for HDLcholesterol, apo B, and triglycerides, respectively (Fig. 5). The strongest relationship was a negative correlation with serum triglycerides. About 35\% (adjusted $r^{2}$ ) of the variation in serum triglycerides, could be explained by the maximum lipolysis rate. In reference to the FCHL patients (open symbols in Fig. 5), the values for HDL cholesterol were found along the regression line of the controls. The triglyceride values in FCHL, however, were higher than predicted from the regression line between lipolysis rate and serum triglyceride values in the controls (Fig. $5) .8$ of the 10 subjects with FCHL had values above the regression line, as compared to 9 of the 22 control subjects $(P<0.05$ by chi-square analysis). A similar tendency was seen for apo B, with 7 out of 10 FCHL patients above the regression line. This difference was, however, not statistically significant.

A stepwise regression analysis was performed to examine the importance of the lipolysis rate for the variations in serum 

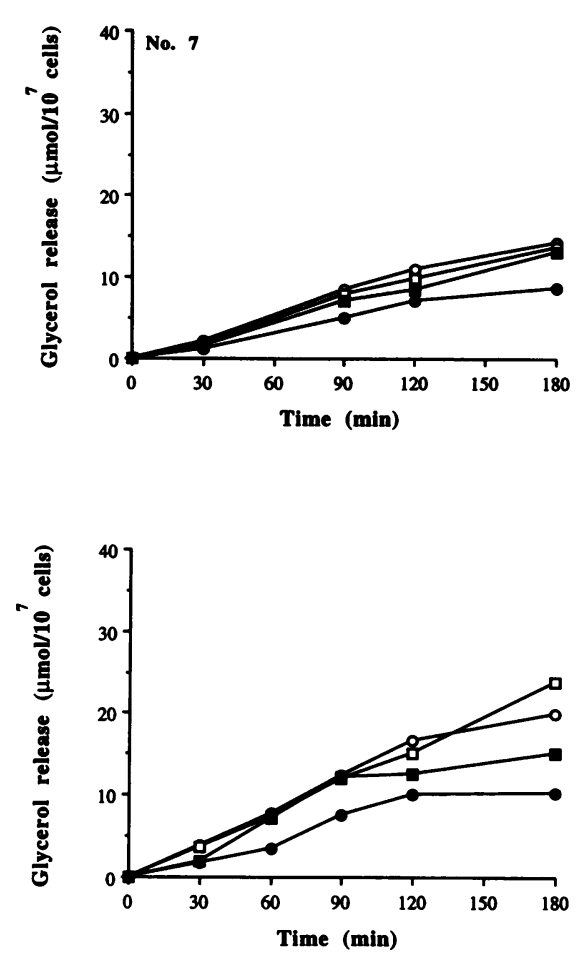
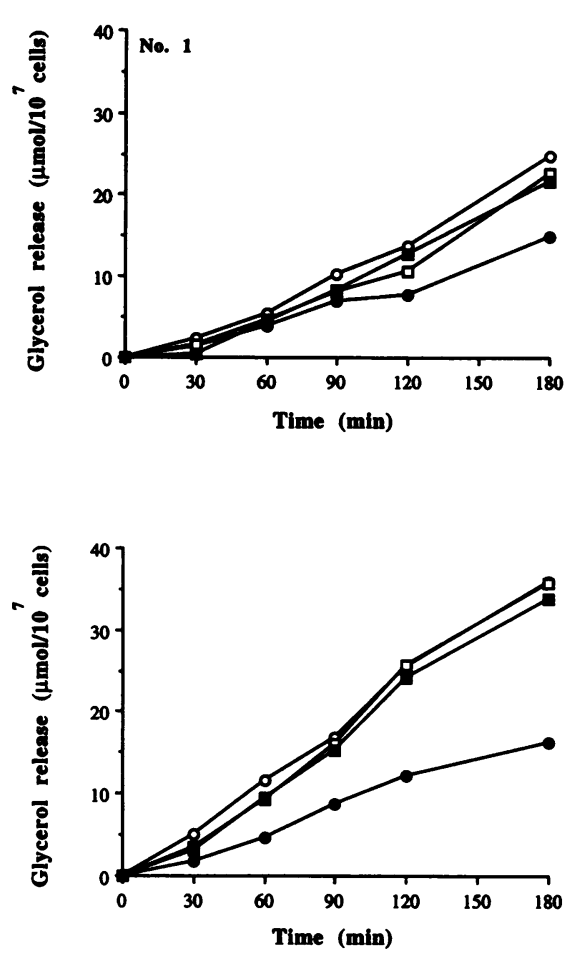

Figure 2. Glycerol release in relation to incubation time in two patients with FCHL (top) and two healthy control subjects (bottom). Isolated fat cells were incubated with maximum effective concentrations of noradrenaline $\left(10^{-7} \mathrm{M}\right.$, filled circles $)$, isoprenaline $\left(10^{-7}\right.$ $\mathrm{M}$, open circles), forskolin ( $10^{-6}$ $\mathbf{M}$, open squares) and dibutyryl cyclic AMP $\left(10^{-3} \mathrm{M}\right.$, filled squares). Glycerol content in the incubation medium was measured at the indicated time points. The numbers of the two patients correspond to the numbers in Table I. triglyceride values. The equation included the triglyceride values for each subject in the control group as the dependent variable, and as independent parameters the maximum isoprenalinestimulated lipolysis rate and other variables which may influence the triglyceride values: age, BMI, waist-hip ratio, fat cell volume, blood glucose, and plasma insulin. Isoprenaline sensitivity $\left(\mathrm{ED}_{50}\right)$, which correlates with lipid levels in healthy subjects (17), was also included in the equation. The triglyceride values were calculated in their logarithmic form to normalize the distribution of the values. The variable with the highest partial correlation coefficient was entered at each step, until no variable remained with an F-value $\geq 4$. The maximum stimulated lipolysis rate was the most important regressor $(F=11.6$, $r=0.62$ ), which together with body mass index and waist-hip ratio contributed to the variation in triglyceride values.

Since five patients were without lipid-lowering medication, the data were recalculated, comparing these subjects separately with the control group. The results obtained regarding the clini-
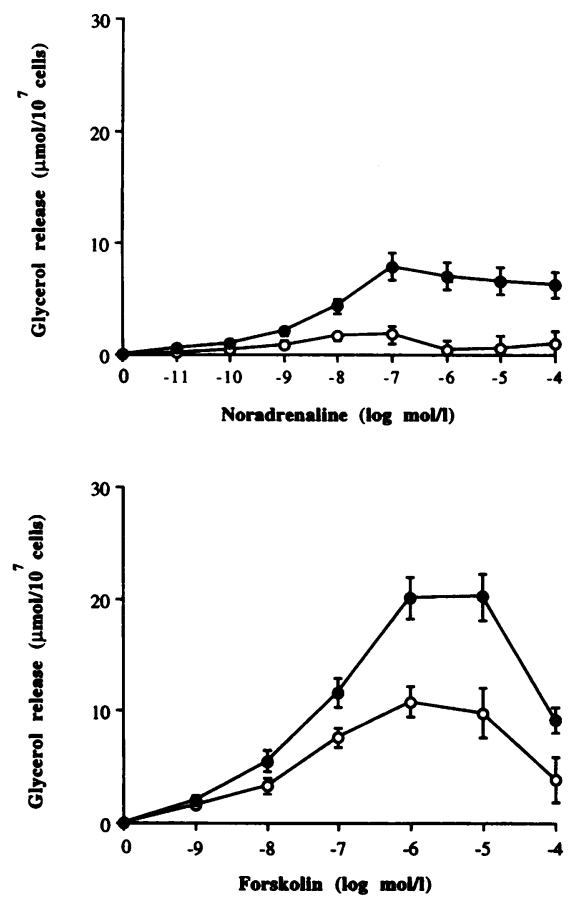
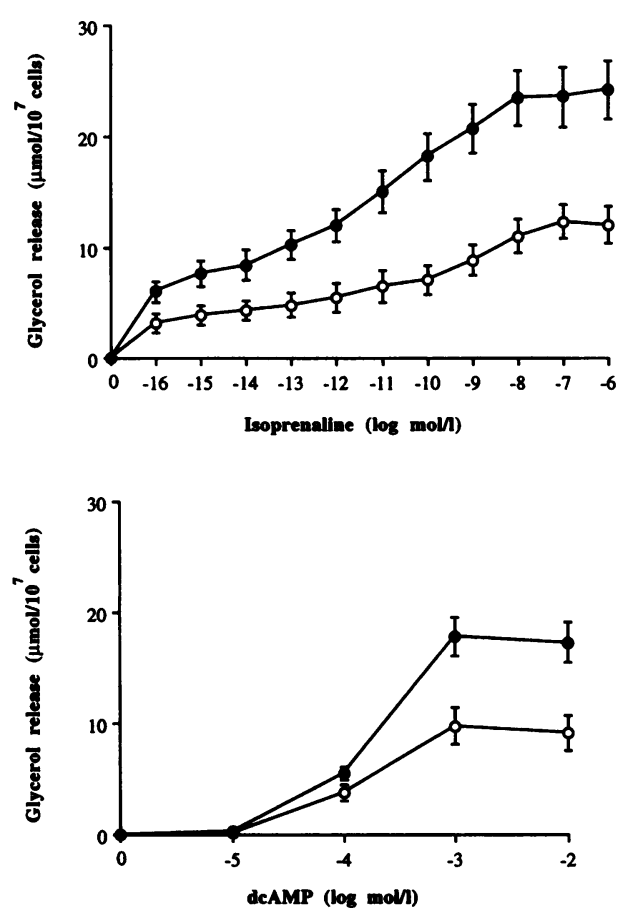

Figure 3. Lipolysis stimulation in FCHL (open symbols) and control subjects (filled symbols). The effect on glycerol release from isolated fat cells of increasing concentrations of noradrenaline, isoprenaline, forskolin, and dcAMP was determined. Mean concentration-response curves are depicted. Values are mean \pm SEM. 
Table III. Maximum Activation of Lipolysis in Isolated Fat Cells

\begin{tabular}{lccc}
\hline & \multicolumn{2}{c}{ Lipolysis rate $(\mu \mathrm{mol} /$ glycerol/107 cells $)$} & \\
\cline { 2 - 3 } & FCHL patients & Control subjects & $P$ \\
\hline Noradrenaline & $3.3 \pm 0.5$ & $8.6 \pm 1.2$ & $<0.01$ \\
Isoprenaline & $12.9 \pm 1.7$ & $25.6 \pm 2.6$ & $<0.005$ \\
Forskolin & $11.6 \pm 1.6$ & $21.9 \pm 2.0$ & $<0.005$ \\
dcAMP & $9.8 \pm 1.7$ & $19.6 \pm 1.7$ & $<0.005$
\end{tabular}

Lipolysis stimulation was determined in the presence of maximum effective agonist concentrations. The values are mean \pm SEM. They were compared using Student's $t$ test.

cal, lipolysis, and radioligand binding data were the same as for the entire group (data not shown).

The possibility that a defect in HSL might cause the observed lipolytic catecholamine resistance was investigated by measuring the maximum activity of this enzyme. The individual data are shown in Fig. 6. The group values ( $\mathrm{mU} / \mathrm{mg}$ protein) were $76 \pm 9$ and $46 \pm 3$ in control and FCHL subjects, respectively $(P=0.029)$. Thus, HSL activity was $40 \%$ decreased in FCHL. In the whole material there was a linear relationship between maximum HSL activity and the maximum lipolysis rate $(r=0.57 ; P<0.001)$. There was, however, no correlation between the enzymatic activity and the serum lipid levels (data not shown).

\section{Discussion}

The present study was undertaken to study lipolysis regulation in FCHL. Even with a relatively strict operative definition (5), as used here, it is possible (and even likely) that FCHL will turn out to represent a mixture of various disease entities, with several metabolic defects. Nevertheless, the present work demonstrates, for the first time, a distinct cellular abnormality in lipolysis regulation in this disorder. Thus, the lipolytic response to catecholamines in adipocytes from patients with FCHL was markedly reduced. This reduction $(\sim 65 \%)$ was not dependent on the use of denominator for lipolysis rates, since similar results were observed when lipolysis rate was expressed per cell number or lipid weight. Furthermore, the reduction was of the same order of magnitude whether lipolysis was stimulated with the adrenergic agonists noradrenaline or isoprenaline, with forskolin which activates the adenylyl cyclase, or with dcAMP which acts directly on the protein kinase-HSL complex. This strongly suggests that the observed defect is localized at the last step in the lipolytic cascade, i.e., the activation of the lipase, since a decrease in the lipolytic action of the nonmetabolizing cAMP analogue (dcAMP) cannot be explained by alterations in lipolysis activation above cAMP. Furthermore, the rate of lipolysis activation was linear for at least $3 \mathrm{~h}$ in both groups, which indicates that the onset of activation of the protein kinase-HSL complex was not altered in FCHL.

The function of the three major human adipocyte adrenergic receptors $\left(\beta_{1}, \beta_{2}\right.$, and $\left.\alpha_{2}\right)$ was normal in FCHL. This is in contrast to previous findings of a correlation between low $\beta_{2}$ receptor function and high circulating lipid levels in healthy subjects (17). This suggests that multiple defects in catecholamine-induced lipolysis of fat cells could be involved in the
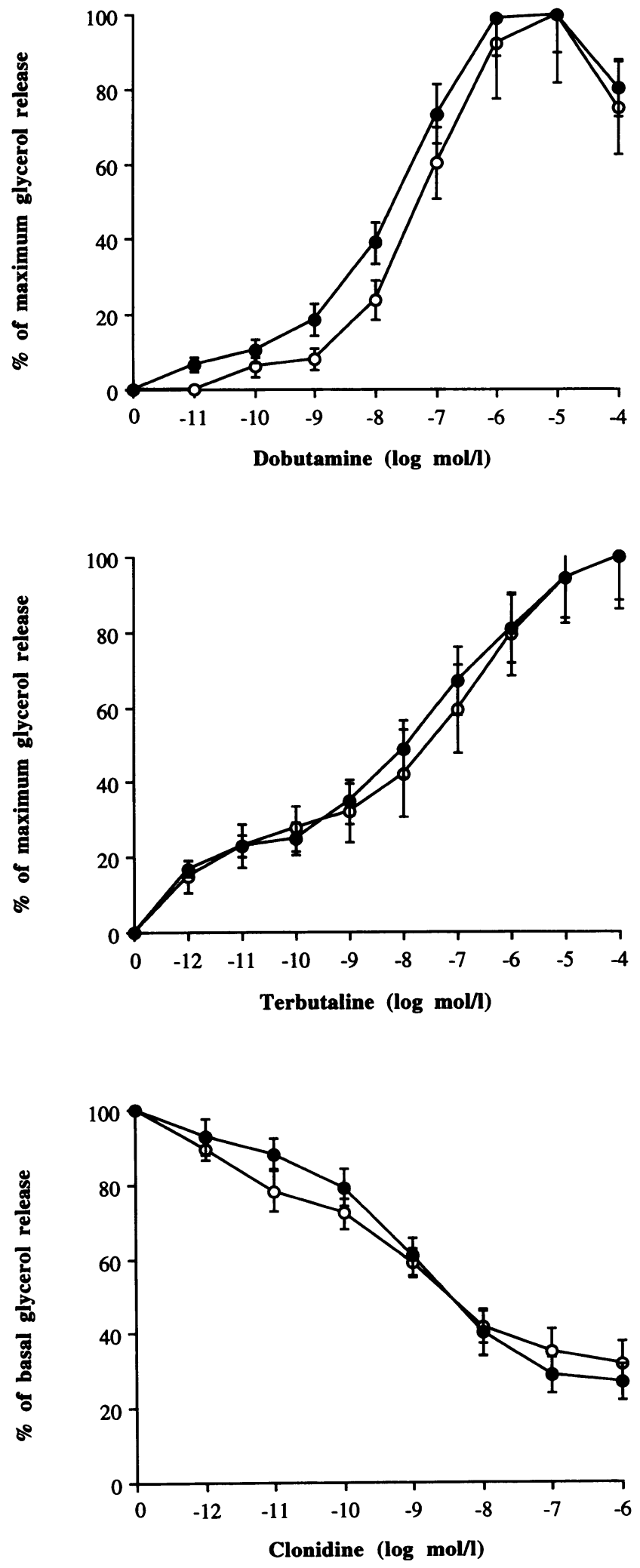

Figure 4. Lipolytic sensitivity to receptor subtype specific agonists in FCHL (open symbols) and control subjects (filled symbols). Lipolysis was stimulated by terbutaline (selective $\beta_{2}$-agonist) or dobutamine (selective $\beta_{1}$-agonist). Mean concentration-response curves are depicted, expressed in percentage of the maximum lipolytic response. Lipolysis was inhibited by clonidine ( $\alpha_{2}$-agonist). Mean dose-response curves are depicted in percentage of the basal rate. Values are mean \pm SEM. 
Table IV. Lipolytic Sensitivity to Selective Adrenergic Agents

\begin{tabular}{lrrr}
\hline & \multicolumn{2}{c}{$\mathrm{ED}_{\text {s0 }}$ (log mol/iter) } & \\
\cline { 2 - 3 } & \multicolumn{1}{c}{ FCHL patients } & \multicolumn{1}{c}{ Control group } & $P$ \\
\hline Noradrenaline & $-7.7 \pm 0.7$ & $-8.3 \pm 0.2$ & NS \\
Isoprenaline & $-12.0 \pm 1.0$ & $-12.4 \pm 0.4$ & NS \\
Terbutaline & $-7.7 \pm 0.4$ & $-8.2 \pm 0.3$ & NS \\
Dobutamine & $-7.3 \pm 0.2$ & $-7.7 \pm 0.2$ & NS \\
Clonidine & $-9.9 \pm 0.3$ & $-9.3 \pm 0.2$ & NS \\
& & & \\
\hline
\end{tabular}

The agonist concentration giving half maximum effect on lipolysis $\left(E_{50}\right)$ was determined from concentration-response curves. The values are mean \pm SEM. They were compared using the unpaired Student's $t$ test.

development of hyperlipidemia. Catecholamine resistance due to a defect in the hormone-sensitive lipase seems, however, to be the most important one for FCHL. It is possible that there are additional early defects in the stepwise lipolysis activation, which may be localized at the level of adenylyl cyclase or cyclic AMP metabolism. Unfortunately, it was not possible to study these events in parallel because the methods require very large amounts of adipose tissue. In any case, such early defects would be of less importance for lipolytic catecholamine resistance in FCHL than the observed defect in hormone-sensitive lipase.

Further strong evidence for a defect in HSL in FCHL are the findings with direct measurement of the enzyme activity in adipose tissue. For this purpose, we used a previously described method for studies of the enzyme activity in human adipose tissue in clinical conditions $(30,31)$. In our hands, the method was as sensitive and reproducible as originally reported. The results show a $40 \%$ decrease in the maximum enzyme activity. In the whole material, the variations in this activity correlated significantly with variations in maximum lipolytic capacity. Thus, it appears that impaired function of HSL is the major (if not only) lipolysis defect in FCHL.

Reduced triglyceride clearance from plasma to peripheral tissues has been suggested by several investigators as a pathophysiological mechanism in FCHL. Hereditary lipoprotein lipase deficiency, demonstrated in a subset of FCHL families (11), may cause reduced peripheral uptake of FFA, and the association of FCHL to the apolipoprotein AI-CIII-AIV gene cluster may also be explained by altered lipoprotein lipase function $(12,13)$. Furthermore, reduced adipocyte triglyceride synthesis due to reduced responsiveness to the acylation-stimulating protein $(16,18-20)$, has been suggested. How do the results of the present study fit with these observations?

One possibility is that the observed reduction in lipolysis rate coexists with a decreased rate of lipid storage in fat cells. As mentioned earlier, this is logical, since, in a simplified model, reduced lipid storage with intact lipolysis function would lead to a depletion of intracellular lipids, and thus a reduction in adipocyte size. Fat cell volume was, however, normal in FCHL. A combined defect in lipid storage and lipolysis would then imply that the adipose tissue is metabolically inactive in FCHL. These events could not, unfortunately be examined simultaneously since the required additional large amount of adipose tissue for a parallel determination of lipid synthesis cannot be obtained in this type of clinical study. At present, it is not possible to conclude which of the defects in lipid synthesis
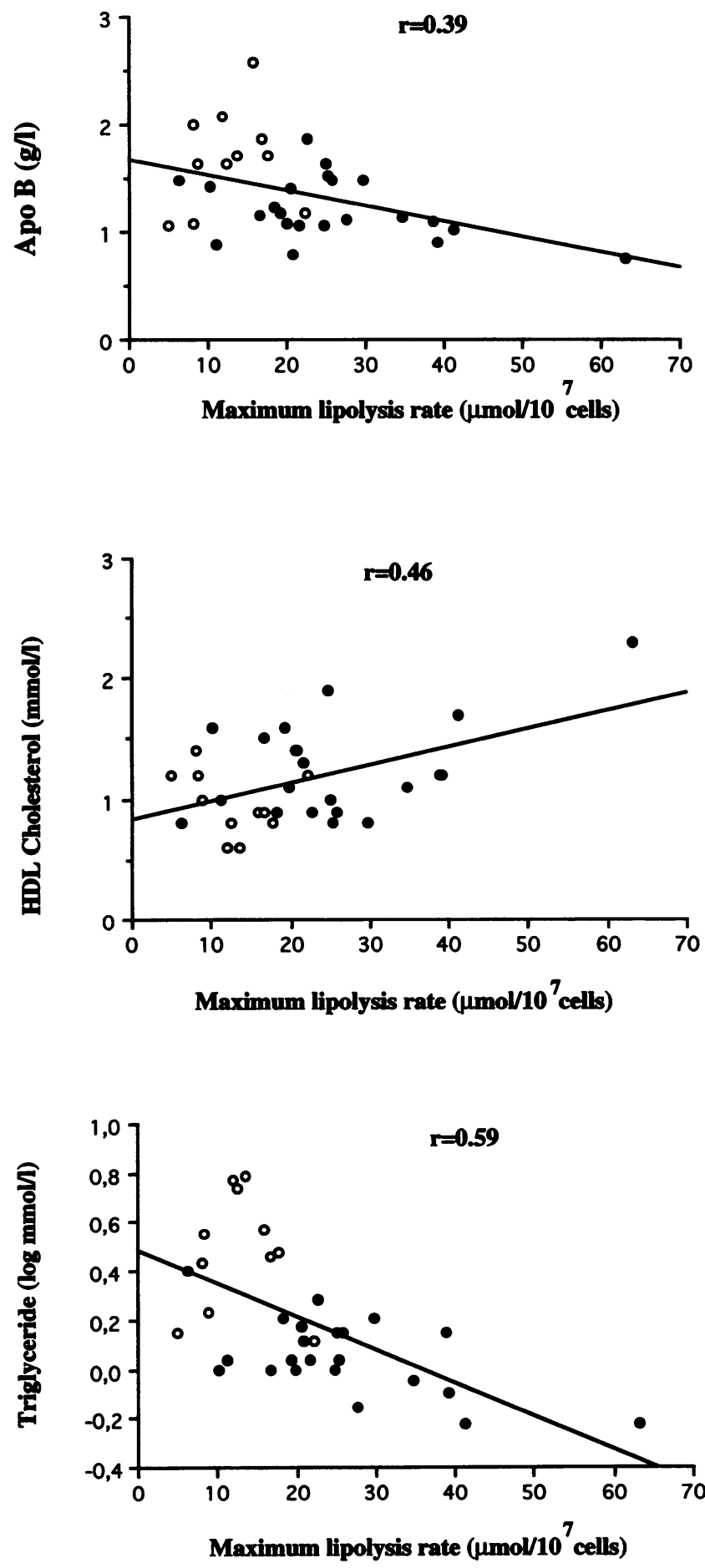

Figure 5. Linear relationship between serum lipid and lipoprotein levels and maximum lipolytic capacity in fat cells from control subjects (filled symbols). The open symbols indicate the values of the $10 \mathrm{FCHL}$ patients in relation to the regression line of the control group. The lipolysis rate was the maximum rate of glycerol release in the presence of isoprenaline (nonselective $\beta$-agonist). Triglyceride values are presented in their logarithmic form.

(previously demonstrated) (16) and breakdown (presently demonstrated) in fat cells is primary or secondary in FCHL. One possible link between these findings is the previous obser- 


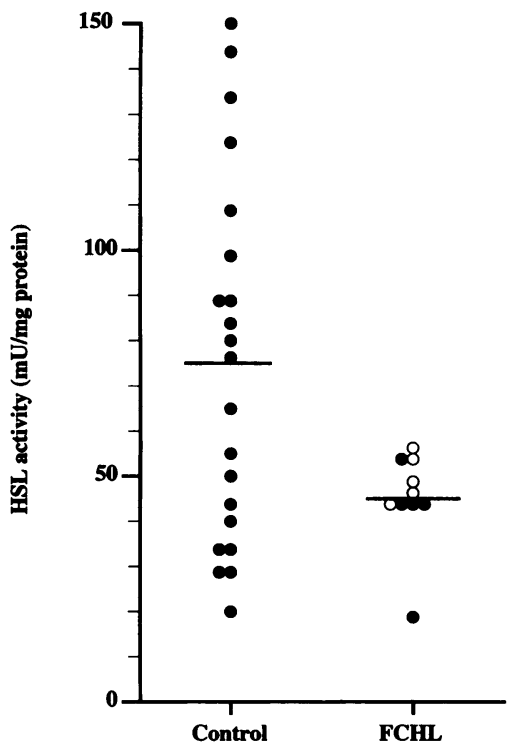

Figure 6. Individual values of maximum hormone-sensitive lipase activity in adipose tissue of control subjects and FCHL patients. FCHL patients on treatment with lipid lowering agents are shown with open symbols. The vertical lines indicate the mean values. The groups were statistically compared by Student's $t$ test $(P<0.05)$.

vation of Edens et al. (34), that newly synthesized diglycerides appear to be more accessible for the hormone-sensitive lipase than the intracellular triglyceride stores. When these previous data are compared with the results of the present study, the diglyceride pathway appears to be of minor importance, however, accounting for $<10 \%$ of the total lipolysis rate. Identification of the primary defect can possibly be achieved through family studies and/or detailed structural and genetic analysis of the HSL.

The lipolysis rate was found to correlate with the serum levels of triglycerides, HDL cholesterol, and apo B. In FCHL, however, the triglyceride levels were higher than predicted from the relationship observed in the controls and did not correlate with the lipolysis rates. This suggests that hypertriglyceridemia and a defective regulation of triglyceride turnover rate in relation to the metabolism of adipose tissue may be a primary metabolic defect in FCHL. A similar tendency was seen regarding the relationship between lipolysis rate and apo B levels; the significance of this observation is uncertain.

Previously, there has been some controversy regarding the classification of FCHL in relation to hyperapobetalipoproteinemia (5). In many cases, these disorders are considered together as different clinical manifestations of a multifactorial disorder $(13,15,20)$. No attempt was made in this study to distinguish between hyperapobetalipoproteinemia and FCHL. All the FCHL patients studied had apo B levels $>1.0 \mathrm{~g} /$ liter, however, and the mean apo B levels were clearly higher in FCHL than in the control group, although there was some overlap in these values between the groups.

The mechanisms behind the increased circulating apo B level in FCHL are largely unknown, but it may be due to hepatic overproduction in response to an increased flux of lipids to the liver, as discussed $(15,20)$. This suggested pathophysiological mechanism for FCHL is also compatible with decreased lipid turnover in fat cells. If the triglyceride pool in subcutaneous adipose tissue is more or less metabolically inactive, FFA and incompletely hydrolyzed chylomicron remnants would be shunted to the liver. Although a rough measure, the slightly higher waist-hip ratio in the FCHL patients as compared to the weight-matched control group indicates a larger visceral fat mass. This fat depot may be of greater importance in FCHL, delivering FFA directly to the liver via the portal circulation. Whether the lipolysis defect found in subcutaneous tissue in the present study is also found in the visceral adipose tissue, or is instead compensated for by an increased lipolysis rate in the visceral fat depot, can only be speculated upon, since visceral adipose tissue is not accessible for the present type of study.

An association with the insulin resistance syndrome has been demonstrated in patients with FCHL $(15,35,36)$. Besides insulin resistance, this syndrome includes upper body obesity, hyperinsulinemia, glucose intolerance, essential hypertension, and dyslipidemia characterized by reduced serum HDL cholesterol and increased serum triglyceride, VLDL, and occasionally LDL cholesterol levels (37). Thus one might speculate that the defect in lipolysis activation found in the present study is caused by chronic insulin-induced inhibition of HSL activity. The FCHL patients did indeed have a significantly higher waist-hip ratio and fasting blood glucose level compared to the age and body weight-matched control subjects. On the other hand, fasting plasma insulin levels were not elevated in the patients with FCHL studied here, which makes an important contribution of hyperinsulinemia and/or insulin resistance for the observed lipolysis defect unlikely.

Catecholamine resistance, due to a defect in cAMP-mediated lipolysis activation, has previously been demonstrated in elderly, apparently healthy men with normal body weight (38). This is interesting, considering that although FCHL is a hereditary disorder, it seldom manifests itself with increased serum lipid levels before $20 \mathrm{yr}$ of age (5). Thus, the lipolysis defect in FCHL resembles a "premature aging" of the fat cells.

Before entering the study, five patients were on treatment with lipid-lowering agents. This reflects the severity of the disorder, most identified patients being on some treatment for coronary heart disease at an early age. However, patients on treatment with drugs with documented effects on lipolysis regulation were not included in the study. For ethical reasons, it was not feasible to remove the medication during the study, but recalculating the data, comparing the untreated patients with the control group, the findings were essentially the same as with the whole group. We therefore assume that the results are representative of males with FCHL. Whether the results also apply to females with FCHL remains to be examined.

In conclusion, the present study demonstrates, for the first time, a defect in lipolysis activation in men with FCHL. This defect is predominantly localized at the final and rate-limiting step of the lipolysis cascade, hormone-sensitive lipase, although additional defects in lipolysis regulation between adrenoreceptors and HSL may be present as well. The lipolysis defect is correlated with the serum lipid abnormalities typical for this disorder. Further studies should now be directed at evaluating the molecular mechanisms and relevance of this defect in the pathogenesis of FCHL, its pattern of inheritance, and its interrelation to other abnormalities observed in this clinically important metabolic disease.

\section{Acknowledgments}

This study was supported by grants from the Swedish Medical Research Council (19X-1034, 03X-7137), the Swedish Diabetes Association, the Karolinska Institute, and the foundations of Osterman, Nordic Insulin, Old Female Servants, Thuring, Golje, Trygg-Hansa, and King Gustaf V. 


\section{References}

1. Goldstein, J. L., H. G. Schrott, W. R. Hazzard, E. L. Bierman, and A. G. Motulsky. 1973. Hyperlipidemia in coronary heart disease. II. Genetic analysis in 176 families and delineation of a new inherited disorder, combined hyperlipidemia. J. Clin. Invest. 52:1544-1568.

2. Rose, H. G., P. Kranz, M. Weinstock, J. Juliano, and J. I. Haft. 1972. Inheritance of combined hyperlipoproteinemia: evidence for a new lipoprotein phenotype. Am. J. Med. 54:148-160.

3. Nikkilä, E. A., and A. Aro. 1973. Family study of serum lipids and lipoproteins in coronary heart disease. Lancet. i:954-958.

4. Brunzell, J. D., H. G. Schrott, A. G. Motulsky, and E. L. Bierman. 1976. Myocardial infarction in the familial forms of hypertriglyceridemia. Metab. Clin. Exp. 25:313-320.

5. Grundy, S. M., A. Chait, and J. D. Brunzell. 1987. Familial combined hyperlipidemia workshop. Arteriosclerosis. 7:203-207.

6. Brunzell, J. D., J. J. Albers, A. Chait, S. M. Grundy, E. Groszek, and G. B. McDonald. 1983. Plasma lipoproteins in familial combined hyperlipidemia and monogenic familial hypertriglyceridemia. J. Lipid Res. 24:147-155.

7. Chait, A., J. J. Albers, and J. D. Brunzell. 1980. Very low density lipoprotein overproduction in genetic forms of hypertriglyceridaemia. Eur. J. Clin. Invest. 10:17-22.

8. Janus, E. D., A. M. Nicoll, P. R. Turner, P. Magill, and B. Lewis. 1980. Kinetic basis of the primary hyperlipidaemias: studies of apolipoprotein B turnover in genetically defined subjects. Eur. J. Clin. Invest. 10:161-172.

9. Kissebah, A. H., S. Alfarsi, and P. W. Adams. 1981. Integrated regulation of very low density lipoprotein triglyceride and apolipoprotein-B kinetics in man: normolipemic subjects, familial hypertriglyceridemia and familial combined hyperlipidemia. Metab. Clin. Exp. 30:856-868.

10. Angelin, B., K. S. Hershon, and J. D. Brunzell. 1987. Bile acid metabolism in hereditary forms of hypertriglyceridemia: evidence for an increased synthesis rate in monogenic familial hypertriglyceridemia. Proc. Natl. Acad. Sci. USA. 84:5434-5438.

11. Babirak, S. P., P.-H. Iverius, W. Y. Fujimoto, and J. D. Brunzell. 1989. Detection and characterization of the heterozygote state for lipoprotein lipase deficiency. Arteriosclerosis. 9:326-334.

12. Hayden, M. R., H. Kirk, C. Clark, J. J. Frohlich, S. W. Rabkin, R. McLeod, and J. Hewitt. 1987. DNA polymorphisms in and around the apo A I-C III gene and genetic hyperlipidemias. Am. J. Hum. Genet. 40:421-430.

13. Wojciechowski, A. P., M. Farrall, P. Cullen, T. M. E. Wilson, J. D. Bayliss, B. Farren, B. A. Griffin, M. J. Caslake, C. J. Packard, J. Shepherd, et al. 1991. Familial combined hyperlipidemia linked to the apolipoprotein AI-CIIIAIV gene cluster on chromosome 11q23-q24. Nature (Lond.). 349:161-164.

14. Castro Cabezas, M., T. W. A. de Bruin, L. A. W. Kock, W. Kortlandt, M. van Linde-Sibenius-Trip, H. Jansen, and D. W. Erkelens. 1993. Simvastatin improves chylomicron remnant removal in familial combined hyperlipidemia without changing chylomicron conversion. Metab. Clin. Exp. 42:497-503.

15. Castro Cabezas, M., T. W. A. de Bruin, H. W. de Valk, C. C. Shoulders, H. Jansen, and D. W. Erkelens. 1993. Impaired fatty acid metabolism in familial combined hyperlipidemia. A mechanism associating hepatic apoliporotein B overproduction and insulin resistance. J. Clin. Invest. 92:160-168.

16. Teng, B., A. Forse, A. Rodriguez, P. O. Kwiterovich, Jr., and A. D. Sniderman. 1988. Adipose tissue triglyceride synthesis in patients with hyperapobetalipoproteinemia. J. Physiol. Pharmacol. 66:239-242.

17. Arner, P., H. Wahrenberg, F. Lönnqvist, and B. Angelin. 1993. Adipocyte $\beta$-adrenoceptor sensitivity influences plasma lipid levels. Arterioscler. Thromb. 13:967-972.

18. Cianflone, K., M. H. Maslowska, and A. D. Sniderman. 1990. Impaired response of fibroblasts from patients with hyperapobetalipoproteinemia to acylation-stimulating protein. J. Clin. Invest. 85:722-730.

19. Baldo, A., A. D. Sniderman, S. St.-Luce, R. K. Avramoglu, M. Maslowska B. Hoang, J. C. Monge, A. Bell, S. Mulay, and K. Cianflone. 1993. The apdisinacylation stimulating protein system and regulation of intracellular triglyceride synthesis. J. Clin. Invest. 92:1543-1547.

20. Sniderman, A. D., B. G. Brown, B. F. Stewart, and K. Cianflone. 1992 From familial combined hyperlipidemia to hyperapoB: unravelling the overproduction of hepatic apolipoprotein B. Curr. Opin. Lipidol. 3:137-142.

21. Lafontan, M., and M. Berlan. 1993. Fat cell adrenergic receptors and the control of white and brown fat cell function. J. Lipid Res. 34:1057-1091.

22. Ericsson, S., M. Eriksson, L. Berglund, and B. Angelin. 1992. Metabolism of plasma low density lipoproteins in familial combined hyperlipidemia: effect of acipimox therapy. J. Intern. Med. 232:313-320.

23. Arner, P., O. Arner, and J. Östman. 1973. The effect of local anaesthetic agents on lipolysis by human adipose tissue. Life Sci. 13:161-169.

24. Lönnqvist, F., H. Wahrenberg, L. Hellström, S. Reynisdottir, and P. Arner. 1992. Lipolytic catecholamine resistance due to decreased $\beta_{2}$-adrenoceptor expression in fat cells. J. Clin. Invest. 90:2175-2186.

25. Henriksson, P., B. Angelin, and L. Berglund. 1992. Hormonal regulation of serum Lp(a) levels. Opposite effects after estrogen treatment and orchidectomy in males with prostatic carcinoma. J. Clin. Invest. 89:1166-1171.

26. Rodbell, M. 1964. Metabolism of isolated fat cells. I. Effects of hormones on glucose metabolism and lipolysis. J. Biol. Chem. 239:375-380.

27. Hirsch, J., and E. Gallian. 1968. Methods for determination of adipose cell size and number in man and animals. J. Lipid Res. 9:110-119.

28. Kather, H., E. Wieland, A. Scheurer, G. Vogel, U. Widenberg, and C. Joost. 1987. Influences of variation in total energy intake and dietary composition on regulation of fat cell lipolysis in ideal-weight subjects. J. Clin. Invest. 80:566572.

29. Hellmér, J., P. Arner, and A. Lundin. 1989. Automatic luminometric kinetic assay of glycerol for lipolysis studies. Anal. Biochem. 177:132-137.

30. Fredriksson, G., P. Strålfors, N. Ö. Nilsson, and P. Belfrage. 1981. Hormone-sensitive lipase from rat. Methods. Enzymol. 71:636-646.

31. Frayn, K. N., D. Langin, C. Holm, and P. Belfrage. 1993. Hormonesensitive lipase: quantitation of enzyme activity and mRNA in small biopsies of human adipose tissue. Clin. Chim. Acta. 216:183-189.

32. Scatchard, G. 1948. The attractions of proteins for small molecules and ions. Ann. NY Acad. Sci. 51:660-672.

33. Tate, K. M., M. M. Briend-Sutren, L. J. Emorine, C. Delavier-Klutchko, S. Marullo, and A. D. Strosberg. 1991. Expression of three human $\beta$-adrenergic receptor subtypes in transfected chinese hamster ovary cells. Eur. J. Biochem. 196:357-361.

34. Edens, N. K., R. L. Leibel, and J. Hirsch. 1990. Lipolytic effects on diacylglycerol accumulation in human adipose tissue in vitro. J. Lipid Res. 31:1351-1359.

35. Williams, R. R., S. C. Hunt, P. N. Hopkins, B. M. Stults, L. L. Wu, S. J. Hasstedt, G. K. Barlow, S. H. Stephenson, J. M. Lalouel, and H. Kouda. 1988. Familial dyslipidemic hypertension: evidence from 58 Utah families for a syndrome present in approximately $12 \%$ of patients with essential hypertension. JAMA (J. Am. Med. Assoc.). 259:3579-3586.

36. Hunt, S. C., L. L. Wu, P. N. Hopkins, B. M. Stults, H. Kouda, M. E. Ramirez, J.-M. Lalouel, and R. R. Williams. 1989. Apolipoprotein, low density lipoprotein subfraction, and insulin associations with familial combined hyperlipidemia. Study of Utah patients with familial dyslipidemic hypertension. Arteriosclerosis. 9:335-344.

37. DeFronzo, R. A. 1992. Insulin resistance, hyperinsulinemia, and coronary artery disease: a complex metabolic web. Coron. Artery Dis. 3:11-25.

38. Lönnqvist, F., B. Nyberg, H. Wahrenberg, and P. Arner. 1990. Catecholamine induced lipolysis in adipose tissue of the elderly. J. Clin. Invest. 85:16141621. 